\title{
Inpatient Medication Reconciliation at Admission and Discharge: A Retrospective Cohort Study of Age and Other Risk Factors for Medication Discrepancies
}

\author{
Kathleen Tschantz Unroe, MD, MHA ${ }^{1,2}$, Trista Pfeiffenberger, MS, PharmD ${ }^{3}$, Sarah \\ Riegelhaupt, PharmD ${ }^{3}$, Jennifer Jastrzembski, PharmD ${ }^{4}$, Yuliya Lokhnygina, PhD $^{1}$, and \\ Cathleen Colón-Emeric, MD ${ }^{1,2}$ \\ ${ }^{1}$ Duke University Medical Center, Durham, North Carolina \\ ${ }^{2}$ Geriatric Research, Education and Clinical Center, Durham VA Medical Center, Durham, North \\ Carolina \\ ${ }^{3}$ Department of Pharmacy, University of North Carolina at Chapel Hill, Chapel Hill, North Carolina \\ ${ }^{4}$ Department of Pharmacy, Monroe Carell Jr. Children's Hospital at Vanderbilt, Nashville, \\ Tennessee
}

\begin{abstract}
Background-Medication discrepancies are unintended differences between medication regimens (ie, between a patient's home regimen and medications prescribed on admission to the hospital).

Objective-The goal of this study was to describe the incidence, drug classes, and probable importance of hospital admission medication discrepancies and discharge regimen differences, and to determine whether factors such as age and specific hospital services were associated with greater frequency of medication discrepancies and differences.

Methods-This was a retrospective cohort study of a random sample of adult patients admitted to the general medicine, cardiology, or general surgery services of a tertiary care academic teaching hospital between July 1, 2006, and August 31, 2006. A chart review was performed to collect the following information: patient demographic characteristics, comorbid conditions, number of preadmission medications, discrepant medications identified by the hospital's reconciliation process, reasons for the discrepancies, and discharge medications that differed from the home regimen. Potentially high-risk discrepancies and differences were identified by determining if the medications were included on either the Institute for Safe Medication Practices high-alert list or the North Carolina Narrow Therapeutic Index list. Univariate and multivariate logistic regression analyses were used to identify factors associated with medication discrepancies and differences.
\end{abstract}

Results-Of the 205 patients (mean age, 59.9 years; 116 men, 89 women; 60\% white) included in the study, 27 did not have any medications recorded on admission. Of the 178 patients who did have medications listed, 41 had $\geq 1$ discrepancy identified by the reconciliation process on admission $(23 \%$; $95 \%$ CI, 17-29); $19 \%$ (95\% CI, 11-31) of these medications were considered to be potentially high risk. In the multivariate logistic regression model, age (odds ratio [OR] per 5-

(C) 2010 Excerpta Medica Inc. All rights reserved.

Address correspondence to: Kathleen Tschantz Unroe, MD, MHA, Geriatric Research, Education and Clinical Center, Durham VA Medical Center, 508 Fulton Street, Durham, NC 27705. kathleen.unroe@ duke.edu.

The authors have indicated that they have no other conflicts of interest regarding the content of this article. 
year increase $=1.16 ; 95 \% \mathrm{CI}, 1.01-1.33 ; P=0.035)$, presence of high-risk medications on admission $(\mathrm{OR}=76.68 ; 95 \% \mathrm{CI}, 9.13-643.76 ; P<0.001)$, and general surgery service $(\mathrm{OR}=$ $3.31 ; 95 \% \mathrm{CI}, 1.40-7.87 ; P<0.007)$ were associated with a higher proportion of patients with discrepancies on admission. At discharge, 196 patients (96\% [95\% CI, 93-98]) had $\geq 1$ medication change from their home regimen, with 1102 total differences for 205 patients. Less than half (44\% [95\% CI, 37-51]) of these patients were explicitly alerted at discharge to new medications or dose changes; $12 \%$ (95\% CI, 7-18) were given written instructions to stop taking discontinued home medications. Cardiovascular drugs were the most frequent class involved at both admission (31\%) and discharge (27\%) in medication discrepancies or differences.

Conclusions-Medication discrepancies on admission and medication differences at discharge were prevalent for adult patients admitted to the general medicine, cardiology, and general surgery services in this academic teaching hospital. Medication reconciliation processes have a high potential to identify clinically important discrepancies for all patients.

\section{Keywords}

medication reconciliation; medication discrepancies; transitions of care; patient safety

\section{INTRODUCTION}

Medications are a critical tool in the practice of modern medicine; one third of Americans take $\geq 5$ medications. ${ }^{1}$ When patients enter the hospital setting, medications may be temporarily withheld, new medications may be added to treat acute processes, and chronic medications may be changed. Hospitalized patients are thus at high risk for experiencing abrupt medication changes and errors that may result in adverse drug events (ADEs). Medication discrepancies are unintended differences between medication regimens (ie, between a patient's home regimen and medications prescribed on admission to the hospital). These discrepancies range in severity and may include omission of medications, wrong medication name, and incorrect dosing. Older patients who are more likely to have chronic conditions, visit more providers, and take a greater number of medications may be at particularly high risk for ADEs related to medication discrepancies.

ADEs are unfortunately both common and costly in the hospital setting. ${ }^{1,2}$ Using previous research, the Institute of Medicine reported that a conservative annual estimate of 400,000 in-hospital preventable ADEs would cost $\$ 3.5$ billion in 2006 dollars. ${ }^{1}$ Increased costs can be due to increases in length of stay or pharmacy and laboratory costs. ${ }^{3,4}$ Reducing preventable ADEs is therefore an important goal for both providers and health care system administrators.

A particularly high-risk time for preventable ADEs is when patients transition from one health care setting to another. Prior studies have found that more than half of general internal medicine patients and patients admitted to acute care services have $\geq 1$ unintended medication discrepancy on admission. ${ }^{5,6}$ Other studies have reported that medication discrepancies, including those more likely to cause potential ADEs, were more common at discharge than on admission. ${ }^{7,8}$ One prospective study of 180 general medicine patients found that $75 \%$ of preventable ADEs occurred at discharge. ${ }^{7}$ A study testing the effectiveness of a multidisciplinary medication reconciliation process found that, before the intervention, there was a mean of 0.5 admission medication discrepancy and 3.3 discharge medication discrepancies per patient. ${ }^{8}$

In addition to the unintended discrepancies between a patient's prior home regimen and hospital discharge orders, failure to adequately communicate the planned medication regimen to patients and other care providers at transition points may lead to ADEs or 
therapeutic failure. ${ }^{5,9-11}$ In a study of patients aged $\geq 65$ years who were recently discharged from the hospital, $14 \%$ had discrepancies between what they were actually taking and what was prescribed at discharge. ${ }^{11}$

Detection and management of medication discrepancies to reduce ADEs are a major focus of patient safety efforts. As part of the National Patient Safety Goals program, the Joint Commission on Accreditation of Healthcare Organizations (JCAHO) issued a mandate in 2005 requiring hospitals to perform medication reconciliation at each transition of care. ${ }^{12}$ According to the JCAHO, a process should be in place for comparing the patient's present medications with those ordered, and a complete list of the patient's medications must be communicated to the next provider of care. Furthermore, a complete list of medications should be provided to the patient at discharge. However, in recognition of the difficulties that many organizations are having in meeting these requirements, the JCAHO decided in February 2009 not to consider medication reconciliation in accreditation decisions until the policy was reviewed further, although it continues to monitor progress in this area. ${ }^{13}$

Health care organizations have responded to the JCAHO mandate with various approaches to medication reconciliation, including development of forms completed by nurses and confirmed by physicians, the use of case managers to perform reconciliation, and tools developed using information technology. ${ }^{8,14-21}$ Although data on the effectiveness of these various options are limited, some of the programs have successfully reduced the number and severity of discrepancies using an interdisciplinary process. ${ }^{8,18}$ Some have even found reductions in rehospitalization rates when using medication reconciliation in addition to other tools. ${ }^{22-24}$ Dedhia et $\mathrm{al}^{22}$ tested a geriatric-specific discharge planning tool that included medication reconciliation and found that fewer intervention patients required a return emergency department visit or hospital readmission compared with a preintervention group ( $3 \%$ vs $10 \%$; odds ratio $[\mathrm{OR}]=0.25 ; 95 \% \mathrm{CI}, 0.10-0.62)$. Another study also assessed elderly inpatients assigned to a discharge planning and education intervention program that included medication reconciliation and counseling, in addition to a follow-up phone call after discharge. They reported fewer emergency department visits and readmissions at 30 days versus a control group $(10.0 \%$ vs $38.1 \% ; P=0.04){ }^{24}$

To contribute to the growing literature on medication discrepancies and the reconciliation process, the present study was designed to describe the frequency of discrepancies in admission medications, the medication classes involved, and the factors associated with these discrepancies. All differences between admission and discharge medication lists were recorded, and the manner in which these were communicated to patients was examined. The goal was to determine whether age and specific hospital services were associated with a higher risk of medication discrepancies or differences. We identified which of these might be considered "higher risk" based on the medication class involved. In addition, the discharge list of medications given to patients and the dictated discharge summaries for the next provider of care were compared, with the aim of identifying unmet communication and education needs.

\section{PATIENTS AND METHODS}

\section{Medication Reconciliation Process Admission}

For the purposes of this study, medication discrepancies were defined as any changes in a patient's previous medications that were identified by an inpatient pharmacist as unintended and potentially clinically relevant at the time of the patient's admission. For example, the omission of furosemide in a patient admitted for dehydration would be considered an intended medication change and would not be considered a discrepancy for this analysis. Pharmacists recorded if the medication had been omitted or was the wrong dose or 
frequency in many cases, but if the medication was simply listed as discrepant without further description, it was categorized as not specified.

This study was conducted at Duke University Medical Center (Durham, North Carolina). At this academic teaching hospital, pharmacist medication reconciliation on admission had been initiated in January 2006, which was before the start of our study. The policy was that, within 48 hours of admission, a clinical pharmacist reviewed the patient medication histories obtained by the provider and the nurse, and compared them with medications prescribed on admission for the patient. Approximately 25 clinical pharmacists performed medication reconciliation throughout the hospital. Front-line managers of clinical pharmacist staff provided education and training regarding the medication reconciliation process as established in-hospital policy. Each pharmacist was provided with a hard copy of the manual, which included relevant algorithms and documents describing the medication reconciliation procedure. In addition to medication reconciliation, these pharmacists assessed and recommended appropriate, evidence-based medication therapies for patients, evaluated and reported ADEs, and provided pharmacokinetic and/or drug-specific monitoring.

When discrepancies were found, the pharmacist investigated further by talking with the patient, the patient's caregiver, the admitting physician, or the referring physician, or by calling the patient's pharmacy. The pharmacist reviewed the physician-completed history and physical for documentation of intentional differences between preadmission medications and those started on admission (ie, medications held in antici- pation of surgery), and these were not counted as discrepancies. Discrepancies that were judged by the pharmacist to be clinically relevant were noted in the patient's chart. The pharmacist developed a reconciled preadmission medication list and resolved any discrepancies with admission orders with the prescribing physician. Through this process, all discrepancies were brought to the attention of the inpatient provider and resolved after discussion with the pharmacist. Thus, for admission, the medication reconciliation performed by the inpatient pharmacists was used as part of the hospital's standard process.

\section{Discharge}

The hospital had not yet instituted a discharge medication reconciliation process at the time of this study. For discharge, the investigators performed the reconciliation by comparing admission and discharge medication lists. Because this was done retrospectively, it was not possible to determine how many changes were unintended versus intended, and they were noted as differences. Any inconsistencies in medication lists between the discharge papers given to patients and the dictated discharge summaries for providers were considered unintended and recorded as discrepancies because these 2 lists should have been identical.

\section{Patient Selection}

A retrospective chart review was conducted for a random sample of adult patients admitted to the general medicine, cardiology, or general surgery services of a 954-bed tertiary care academic teaching hospital between July 1, 2006, and August 31, 2006. These 3 ser- vices were chosen because of their high volume and broad patient population. The charts were randomly selected, using a computer-generated random number list, from a record of all patients admitted during the study period. Patients initially admitted to an intensive care unit or transferred from an outside hospital were excluded because admission medication reconciliation should already have occurred. 


\section{Chart Abstraction}

Medical records were abstracted into a Microsoft Access database (Microsoft Corporation, Redmond, Washington) by 2 of the investigators, a physician (K.T.U.) and a pharmacy student (S.R.). Both paper records (admission history from the medical team, preadmission medication list from the nursing team, pharmacist medication reconciliation note, and patient discharge instruction sheet) and electronic records (discharge summary) were used. Reliability was ensured by having both investigators independently review a random 10\% sample of patient charts and demonstrate that simple agreement exceeded $90 \%$. All questions that arose in interpreting data were resolved by consensus during study team meetings.

\section{Variables}

Information including patient demographic characteristics, comorbid conditions, and number of medications was abstracted from the admission records. Discrepancies on admission and differences at discharge were recorded as described earlier.

Written discharge instructions were also examined for instructions regarding the medication differences (eg, whether the patient was explicitly instructed to stop or change the dose of a previous home medication that had been discontinued or dose adjusted). Patient discharge instructions were also compared with dictated discharge summaries, which are routinely sent to follow-up care providers, and any inconsistencies between these 2 lists of medications were recorded as discrepancies.

Potentially high-risk admission discrepancies and discharge differences were identified by determining if the medications were included on the Institute for Safe Medication Practices (ISMP) high-alert list or the North Carolina Narrow Therapeutic Index (NTI) list (Table I) ${ }^{25,26}$ The medications on these lists have a higher risk of patient harm, a higher risk of subtherapeu-tic and supratherapeutic drug concentrations, or both.

\section{Statistical Analysis}

Baseline characteristics were compared among services using the Wilcoxon signed rank test for continuous variables, and the Pearson $\chi^{2}$ test or the Fisher exact test (when expected cell count was $<5$ ) for categorical variables. The data were analyzed using descriptive statistics to determine, overall and by admitting service, the proportion of patients with $\geq 1$ admission discrepancy identified, the proportion of medications by classes that were discrepant, and the types of discrepancies. The proportion of potentially high-risk discrepant medications, overall and by admitting service, was estimated using logistic regression, accounting for within-patient correlation between medications. Similar analyses were performed to assess discharge medication differences. Univariate and multivariate logistic regression analyses were conducted where the response variable was presence or absence of any medication discrepancies for admission and differences for discharge. The variables considered for inclusion in both admission and discharge models were age, sex, race, number of co- morbid conditions, admitting service, number of pre-admission medications, and presence of preadmission high-risk medications; length of hospital stay was additionally considered for the discharge model.

This study was approved by the institutional review board of Duke University Medical Center. 


\section{RESULTS}

A total of 205 patients (mean age, 59.9 years; 116 men, 89 women; $60 \%$ white) were included in the study. Age, sex, and race distribution did not differ statistically among the 3 services (Table II). Cardiology patients had a higher mean number of comorbid conditions (5.2), followed by general medicine patients $(4.0)(P<0.001)$. General surgery patients had the longest mean length of stay (5.4 days) $(P=0.002)$.

\section{Admission}

Of the 205 patients in the study, 27 did not have any medications recorded on admission. Of the 178 patients who did have medications listed, 41 had $\geq 1$ discrepancy noted $(23 \%$; $95 \%$ CI, 17-29). On the cardiology service, 9 patients (15\%; 95\% CI, 6-24) had discrepant medications; 14 general medicine patients (22\%; 95\% CI, 11-32) and 18 general surgery patients (35\%; 95\% CI, 22-49) also had $\geq 1$ medication discrepancy. The proportion of patients with discrepancies on admission differed significantly among the 3 services ( $P=$ $0.03)$.

Of the admission medications that were found to be discrepant, 19\% (95\% CI, 11-31) were considered potentially high risk based on ISMP and NTI classifications. The proportion of high-risk discrepancies was not significantly different among the 3 services.

Cardiovascular medications represented $31 \%(\mathrm{n}=25)$ of all discrepancies on admission, followed by hormone-modifying agents, hematologic/oncologic medications (including chemotherapeutic agents or adjuvant therapies), and central nervous system agents (all, 12\% $[\mathrm{n}=10])$ (Table III). Medication omissions accounted for 37\% ( $\mathrm{n}=30)$ of admission discrepancies, and $4 \%(\mathrm{n}=3)$ were wrong dose or frequency. The type of discrepancy was not specified by the pharmacist in $59 \%$ of cases $(n=48)$.

In the univariate logistic regression analysis of medication discrepancies on admission, presence of high-risk medications ( $\mathrm{OR}=63.14 ; 95 \% \mathrm{CI}, 7.93-502.45)$ and admitting service $(\mathrm{OR}=3.21 ; 95 \% \mathrm{CI}, 1.29-7.98$ for general surgery vs cardiology) were significantly associated with a higher proportion of patients with discrep- ancies $(P<0.001$ and $2 d f, P=$ 0.036 , respectively) (Table IV). In the multivariate logistic regression model, age (OR per 5year increase $=1.16 ; 95 \% \mathrm{CI}, 1.01-1.33 ; P=0.035)$, presence of high-risk medications (OR $=76.68 ; 95 \% \mathrm{CI}, 9.13-643.76 ; P<0.001)$, and general surgery service $(\mathrm{OR}=3.31 ; 95 \% \mathrm{CI}$, $1.40-7.87 ; P<0.007)$ were associated with a higher proportion of patients with discrepancies on admission.

\section{Discharge}

For the 205 patients in this study, 196 (96\%; 95\% CI, 93-98) had $\geq 1$ medication difference (mean, 5.4; range, 0-18) at discharge compared with their admission medications listing. Patients discharged from the cardiology service had 50\% (95\% CI, 43-57) of their medications changed at discharge; general medicine patients had 58\% changed (95\% CI, 51-65) and general surgery patients had 66\% changed (95\% CI, 58-73). There were 1102 total differences at discharge noted for these 205 patients. Cardiovascular medications were the most frequent class involved $(27 \%[\mathrm{n}=298])$ (Table V). Central nervous system, gastrointestinal, antimicrobial, and hematologic/oncologic medications represented 14\% (n $=149), 11 \%(\mathrm{n}=126), 11 \%(\mathrm{n}=121)$, and $9 \%(\mathrm{n}=99)$ of differences, respectively. A total of 560 agents (51\%) were newly added medications, $304(28 \%)$ were discontinued medications, and $118(11 \%)$ were dose changes of previous medications; $120(11 \%)$ were classified as other medication activity. 
Potentially high-risk medications represented $18 \%$ of the discharge differences $(95 \% \mathrm{CI}$, 15-20). Per hospital service, this ranged from $13 \%$ (95\% CI, 10-18) for cardiology, $18 \%$ (95\% CI, 13-23) for general medicine, and 21\% (95\% CI, 17-25) for general surgery. The proportion of high-risk medications was significantly different between admitting services ( $2 d f, P=0.028$ ). The proportion of high-risk medications for cardiology patients was significantly different from that for general surgery patients $(P=0.008)$.

Less than half of the study patients (44\% [95\% CI, 37-51]) were explicitly alerted to new medications or dose changes on their discharge paperwork. Of those patients who had home medications that were discontinued at discharge, $12 \%$ (95\% CI, 7-18) were given written instructions to stop taking them. Such instructions had to be written out in free text on the discharge paperwork by the discharging provider.

Overall, 35\% (95\% CI, 28-41) of patients had discrepancies between the patient discharge paperwork and the dictated discharge summary. By service, 29\% (95\% CI, 18-40) of cardiology patients, 38\% (95\% CI, 27-50) of general medicine patients, and 38\% (95\% CI, 25-50) of general surgery patients had discrepancies between the discharge medication lists (Table VI).

Only the presence of high-risk medication was significantly associated with a higher likelihood of medication differences at discharge $(\mathrm{OR}=9.47$; 95\% CI, 1.12-80.22; $P=$ 0.039), although CIs were wide due to the low prevalence of high-risk medications at admission and the large proportion of patients with $\geq 1$ discharge difference (Table VII).

\section{DISCUSSION}

Hospitals across the country are at various stages of implementation of the JCAHOmandated medication reconciliation at admission and discharge. ${ }^{13}$ This is a resourceintensive process, and it is important to target efforts in ways that maximize the impact on patient safety. In the present study, pharmacists recorded unintended discrepancies between home medication lists and admission medications for $23 \%$ of patients. Many of these discrepancies were in classes of medications that have been previously documented to pose a high risk for ADEs. ${ }^{25,26}$ Our findings are similar to other research on general medicine services, ${ }^{7}$ and we extended them to include cardiology and general surgery services.

We found that nearly all patients (196 of 205) left the hospital with changes in their medication regimens and many did not receive written instructions highlighting these changes (only $12 \%$ of those who had preadmission medications discontinued were explicitly told to stop taking them at discharge). Discrepancies between dictated discharge summaries and written patient instructions are a potential source of confusion for patients and their next provider of care, and there is an urgent need for the development of effective systems to ensure adequate patient education and communication between providers. This will almost certainly require health care systems to allocate additional funds to accomplish this mandate. Present reimbursement systems are not constructed to cover or encourage discharge patient education and communication activities.

There are several important conclusions from this study. First, the process of medication reconciliation has a high potential to identify and correct medication discrepancies in hospitalized patients. Second, while increasing age was associated with discrepancies on admission to the hospital, discrepancies were found across all services and patient groups studied, suggesting that reconciliation efforts cannot be narrowed to high-risk populations based on comorbidities or type of inpatient service. It was surprising that the total number of pre-admission medications was not associated with admission discrepancies. This may be because the pharmacists did not routinely report substances such as vitamins as discrepant 
and also because medications discontinued for a clinical indication were not reported as discrepancies. Our results suggest that preadmission medication class, rather than absolute number of medications, may be the most important predictor of clinically significant medication discrepancies. The association between high- risk medications and discrepancies or differences at both admission and discharge suggests that more intensive reconciliation and education efforts could be targeted toward patients taking these medications. Finally, although efforts in many health care systems have focused on admission medication reconciliation, discharge inconsistencies are particularly prevalent, suggesting that reconciliation, communication, and education efforts at this transition point are urgently needed. ${ }^{27}$

In addition to processes implemented by providers and health care system administrators, some have advocated for the involvement and empowerment of patients and caregivers to reduce ADEs that occur at transitions of care. Coleman et $\mathrm{al}^{28}$ studied a comprehensive approach to helping community-dwelling patients aged $>65$ years through a hospitalization and discharge to home. Interventions for their care transitions included medication reconciliation but also development of a personal health record for the patient and assignment of a transition coach who could both call and visit the patient after discharge. Testing this intervention in a randomized controlled trial of 750 older adults, the researchers found statistically significant reductions in rehospitalization rates at 30 days $(8.3 \%$ vs $11.9 \% ; P=0.048)$ and at 90 days $(16.7 \%$ vs $22.5 \% ; P=0.04)$. A recent study in a teaching hospital in the Netherlands found that medication reconciliation, when coupled with more intensive patient participation and counseling, re- sulted in a more robust process, with a greater number of discrepancies identified and corrected. ${ }^{29}$ In this study, 262 patients (mean [SD] age, 65 [17.3] years) discharged from an inpatient pulmonary ward received medication reconciliation with or without counseling by pharmaceutical consultants. Medication reconciliation was done primarily using electronic medical records of the hospital and pharmacy. Nearly all patients $(97 \%)$ who received counseling had $\geq 1$ additional medication change afterward, either due to another discrepancy that was identified or due to identified therapeutic needs. In addition, patients received personalized counseling and education regarding their medications.

The present study helps illustrate why programs that focus on discharge medication reconciliation can identify and correct these discrepancies. Further studies are needed to determine whether interventions in younger patients, or less resource-intensive interventions, might reduce ADEs and rehospitalization rates. Such interventions could include a follow-up call or single home visit to identify potentially dangerous medication discrepancies. It is possible that proposals ${ }^{30}$ to reimburse hospitals less for readmissions will increase the incentive for the hospital to provide more robust discharge planning, counseling, and follow-up.

The results of the present study may be generalizable to similar academic medical centers with a variety of medical and surgical clinical services that serve a demographically similar population. The primary limitation of this study, however, is that we could not determine what proportion of the corrected medication discrepancies would have resulted in a clinically significant ADE. We infer, based on results of previous research, ${ }^{1}$ that the high number of discrepant and different medications identified creates a significant potential for problems. Although a recent retrospective study of nursing home patients reported that only a small proportion of medication discrepancies resulted in an ADE (65 of 1350), ${ }^{31}$ this study relied on chart review that likely underestimated ADE incidence and severity. Our use of the ISMP and NTI lists alone to identify potentially high-risk discrepancies likely underestimated the clinical significance of many other discrepancies. 
Another limitation of our retrospective study-and medication reconciliation processes in general-is that there is no gold standard for obtaining consistent medication histories from patients. Without an accurate preadmission medication list that reflects what the patient has been taking at home, the entire process is flawed. Our study, however, was intended to examine the real-world implementation of medication reconciliation in our hospital and reflects a standard process for eliciting medication histories by providers. In addition, providers often give oral instructions to patients regarding discharge medications; however, because this is not consistently recorded in the chart, this type of counseling could not be tracked in the present study.

Pharmacist medication reconciliation is an important tool to reduce the risk of medication discrepancies. However, other models of medication reconciliation, such as use of electronic personal health records, should be developed and tested. Patient education about medication regimens and clear communication to the next provider of care are essential for both regulatory compliance and patient safety.

\section{CONCLUSIONS}

Medication discrepancies on admission and medication differences at discharge were prevalent for adult patients admitted to the general medicine, cardiology, and general surgery services in this academic medical center. Medication reconciliation processes have a high potential to identify clinically important discrepancies for all patients.

\section{Acknowledgments}

This work was supported with internal funds from Duke University Medical Center. Dr. Colón-Emeric is supported by Paul A. Beeson Award K23 AG024787.

\section{References}

1. Aspden, P.; Wolcott, JA.; Bootman, JL.; Cronenwett, LR., editors. Committee on Identifying and Preventing Medication Errors. Preventing Medication Errors: Quality Chasm Series. Washington, DC: The National Academies Press; 2007.

2. Bates DW, Cullen DJ, Laird N, et al. for the ADE Prevention Study Group. Incidence of adverse drug events and potential adverse drug events. Implications for prevention. JAMA. 1995; 274:29_ 34. [PubMed: 7791255]

3. Bond CA, Raehl CL, Franke T. Clinical pharmacy services, hospital pharmacy staffing, and medication errors in United States hospitals. Pharmacotherapy. 2002; 22:134-147. [PubMed: 11837551]

4. Classen DC, Pestotnik SL, Evans RS, et al. Adverse drug events in hospitalized patients. Excess length of stay, extra costs, and attributable mortality. JAMA. 1997; 277:301-306. [PubMed: 9002492]

5. Cornish PL, Knowles SR, Marchesano R, et al. Unintended medication discrepancies at the time of hospital admission. Arch Intern Med. 2005; 165:424-429. [PubMed: 15738372]

6. Vira T, Colquhoun M, Etchells E. Reconcilable differences: Correcting medication errors at hospital admission and discharge. Qual Saf Health Care. 2006; 15:122- 126. [PubMed: 16585113]

7. Pippins JR, Gandhi TK, Hamann C, et al. Classifying and predicting errors of inpatient medication reconciliation. J Gen Intern Med. 2008; 23:1414-1422. [PubMed: 18563493]

8. Varkey P, Cunningham J, O’Meara J, et al. Multidisciplinary approach to inpatient medication reconciliation in an academic setting. Am J Health Syst Pharm. 2007; 64:850-854. [PubMed: 17420202]

9. Lessard S, DeYoung J, Vazzana N. Medication discrepancies affecting senior patients at hospital admission. Am J Health Syst Pharm. 2006; 63:740-743. [PubMed: 16595814] 
10. Forster AJ, Murff HJ, Peterson JF, et al. Adverse drug events occurring following hospital discharge. J Gen Intern Med. 2005; 20:317-323. [PubMed: 15857487]

11. Coleman EA, Smith JD, Raha D, Min SJ. Posthospital medication discrepancies: Prevalence and contributing factors. Arch Intern Med. 2005; 165:1842-1847. [PubMed: 16157827]

12. The Joint Commission. [Accessed January 30, 2009] Sentinel Event Alert: Using medication reconciliation to prevent errors. http://www.jointcommission.org/SentinelEvents/ SentinelEventAlert/sea_35.htm

13. Approved: Will not score medication reconciliation in 2009. The Joint Commission plans to review, refine NPSG 8 for 2010. Joint Commission Perspectives. 2009; 29:1-3.

14. Schnipper JL, Hamann C, Ndumele CD, et al. Effect of an electronic medication reconciliation application and process redesign on potential adverse drug events: A cluster-randomized trial. Arch Intern Med. 2009; 169:771-780. [PubMed: 19398689]

15. Clay BJ, Halasyamani L, Stucky ER, et al. Results of a medication reconciliation survey from the 2006 Society of Hospital Medicine national meeting. J Hosp Med. 2008; 3:465-472. [PubMed: 19084896]

16. Stover PA, Somers P. An approach to medication reconciliation. Am J Med Qual. 2006; 21:307309. [PubMed: 16973946]

17. Levanda M. Implementing a medication reconciliation process in a community hospital. Am J Health Syst Pharm. 2007; 64:1372-1378. [PubMed: 17592001]

18. Hayes BD, Donovan JL, Smith BS, Hartman CA. Pharmacist-conducted medication reconciliation in an emergency department. Am J Health Syst Pharm. 2007; 64:1720-1723. [PubMed: 17687061]

19. Poole DL, Chainakul JN, Pearson M, Graham L. Medication reconciliation: A necessity in promoting a safe hospital discharge. J Healthc Qual. 2006; 28:12-19. [PubMed: 17518010]

20. Kramer JS, Hopkins PJ, Rosendale JC, et al. Implementation of an electronic system for medication reconciliation [published correction appears in Am J Health Syst Pharm. 2007;64:684]. Am J Health Syst Pharm. 2007; 64:404-422. [PubMed: 17299180]

21. Wortman SB. Medication reconciliation in a community, nonteaching hospital. Am J Health Syst Pharm. 2008; 65:2047-2054. [PubMed: 18945865]

22. Dedhia $P$, Kravet $S$, Bulger J, et al. A quality improvement intervention to facilitate the transition of older adults from three hospitals back to their homes. J Am Geriatr Soc. 2009; 57:1540-1546. [PubMed: 19694865]

23. Jack BW, Chetty VK, Anthony D, et al. A reengineered hospital discharge program to decrease rehospitalization: A randomized trial. Ann Intern Med. 2009; 150:178- 187. [PubMed: 19189907]

24. Koehler BE, Richter KM, Youngblood L, et al. Reduction of 30-day postdischarge hospital readmission or emergency department (ED) visit rates in high-risk elderly medical patients through delivery of a targeted care bundle. J Hosp Med. 2009; 4:211-218. [PubMed: 19388074]

25. Institute for Safe Medication Practices. [Accessed March 10, 2010] ISMP's List of High-Alert Medications. http://www.ismp.org

26. North Carolina Board of Pharmacy. [Accessed March 23, 2010] North Carolina Narrow Therapeutic Index list. http://ncbop.org/faqs/Pharmacist/faq_NTIDrugs.htm

27. Wong JD, Bajcar JM, Wong GG, et al. Medication reconciliation at hospital discharge: Evaluating discrepancies. Ann Pharmacother. 2008; 42:1373-1379. [PubMed: 18780806]

28. Coleman EA, Parry C, Chalmers S, Min SJ. The care transitions intervention: Results of a randomized controlled trial. Arch Intern Med. 2006; 166:1822-1828. [PubMed: 17000937]

29. Karapinar-Carkit F, Borgsteede SD, Zoer J, et al. Effect of medication reconciliation with and without patient counseling on the number of pharmaceutical interventions among patients discharged from the hospital. Ann Pharmacother. 2009; 43:1001-1010. [PubMed: 19491320]

30. Medicare Payment Advisory Commission. [Accessed March 23, 2010] A path to bundled payment around a hospitalization. http://www.medpac.gov/chapters/Jun08_Ch04.pdf

31. Boockvar KS, Liu S, Goldstein N, et al. Prescribing discrepancies likely to cause adverse drug events after patient transfer. Qual Saf Health Care. 2009; 18:32-36. [PubMed: 19204129] 


\section{Table I}

List of potentially high-risk medications from the Institute for Safe Medication Practices (ISMP) and the North Carolina Narrow Therapeutic Index (NTI). ${ }^{25,26}$

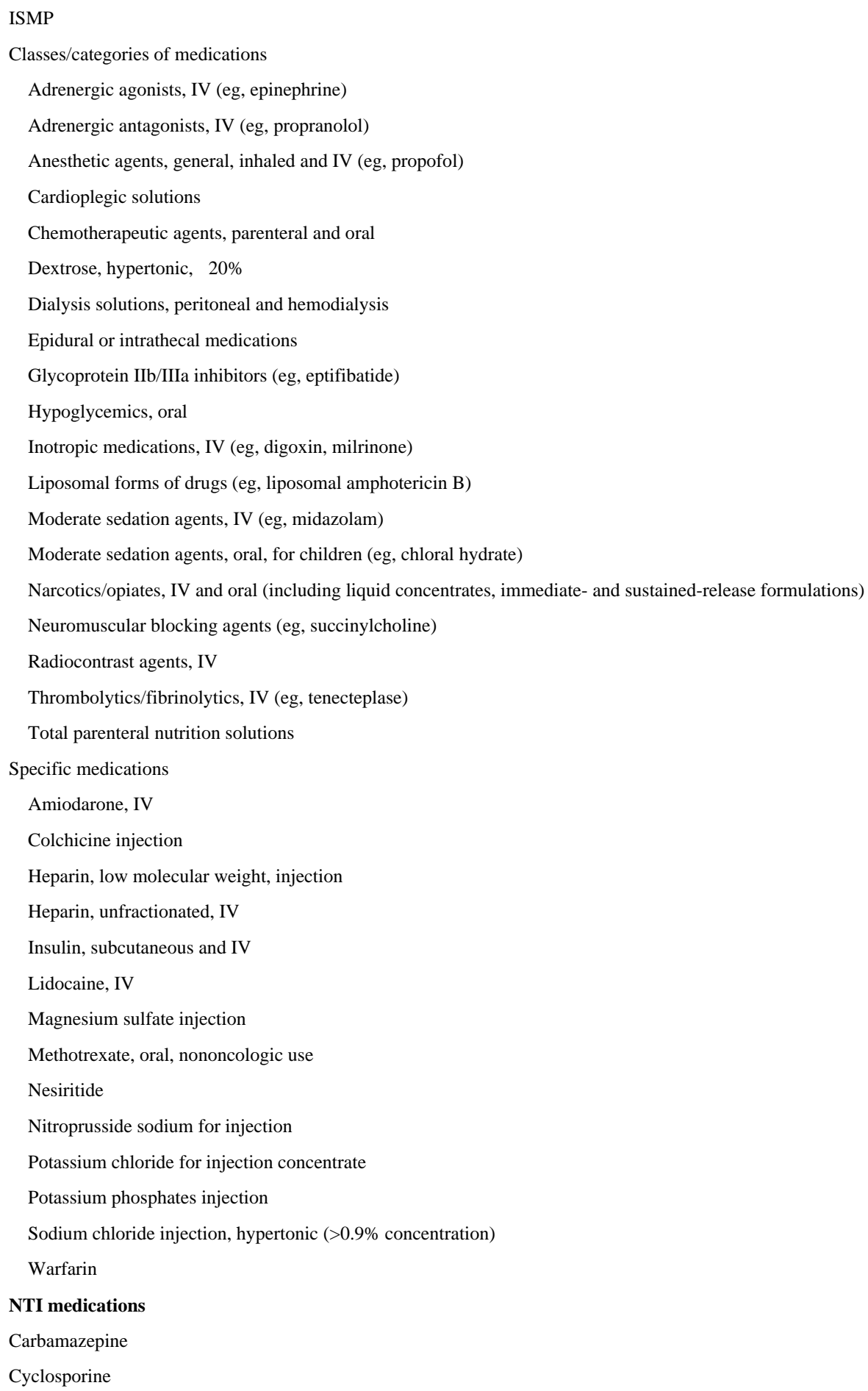




\author{
Digoxin \\ Ethosuximide \\ Levothyroxine \\ Lithium \\ Phenytoin \\ Procainamide hydrochloride \\ Theophylline \\ Warfarin \\ $\mathrm{IV}=$ intravenous.
}


莺

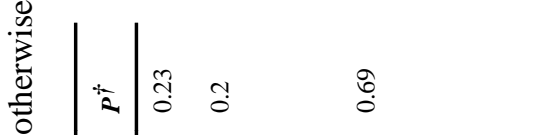

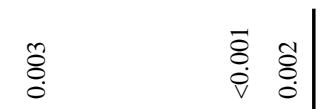

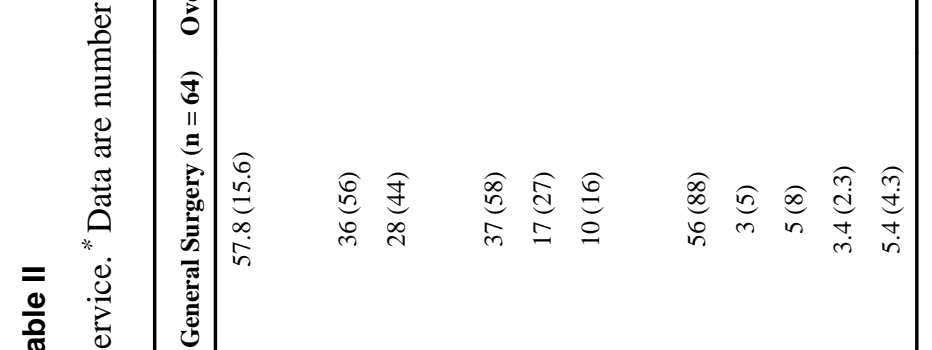

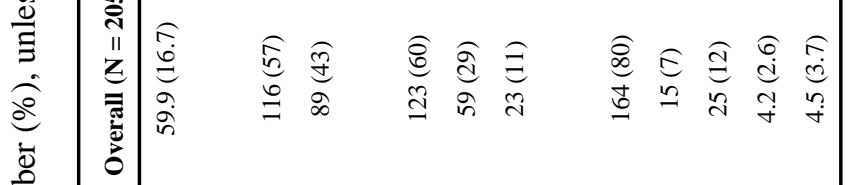

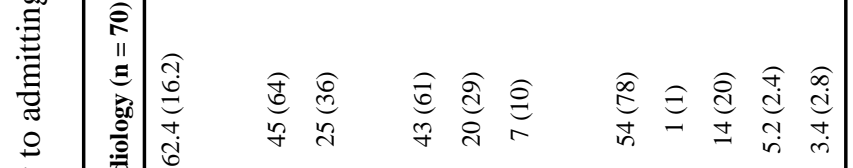

वा

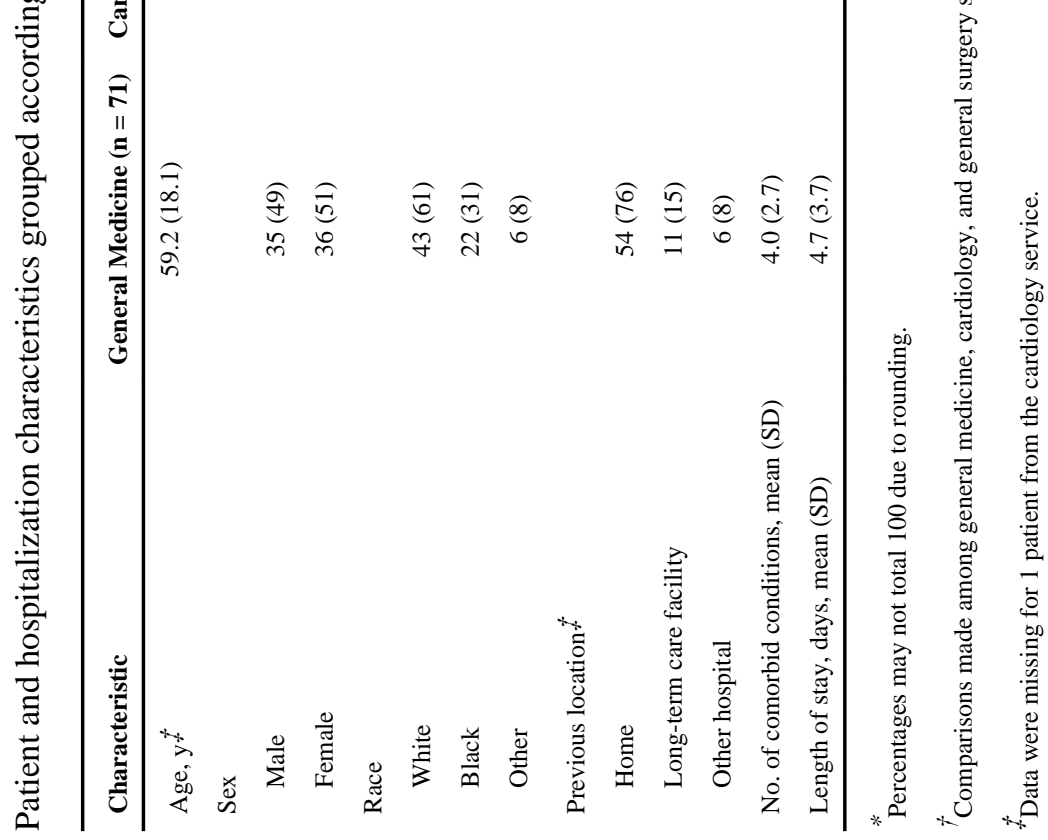

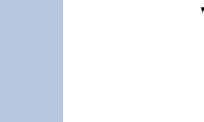

ב

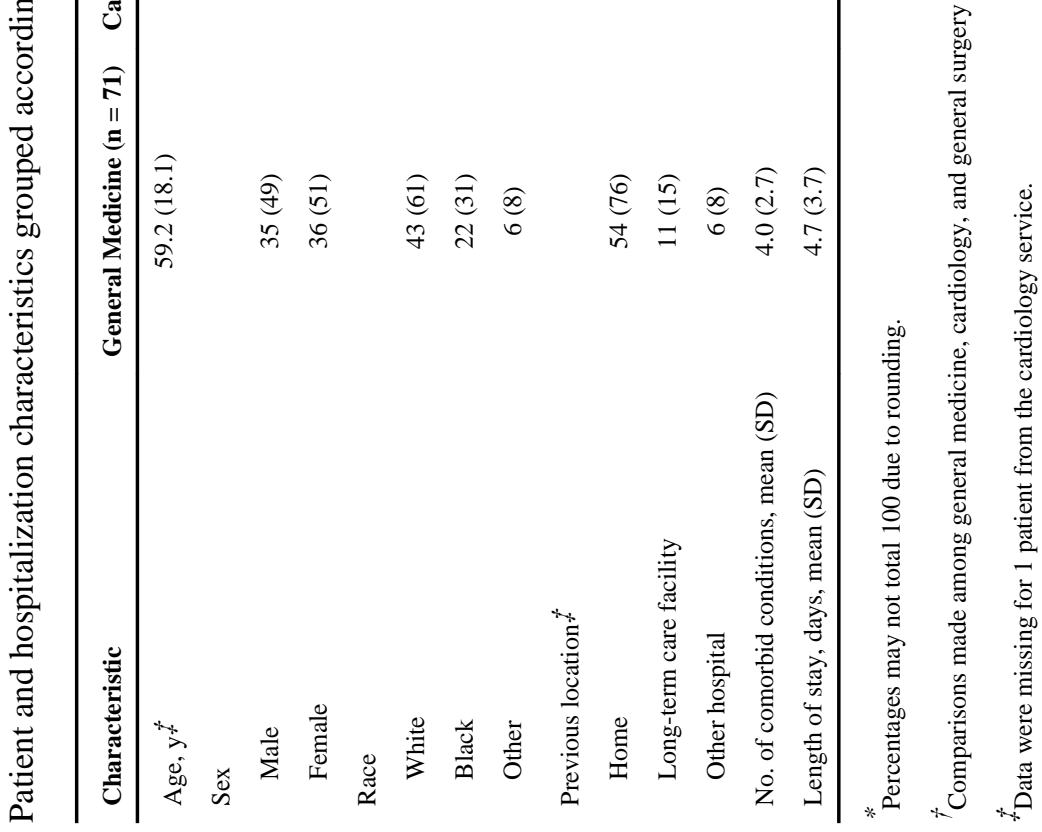

Am J Geriatr Pharmacother. Author manuscript; available in PMC 2013 August 12. 
Table III

Admission medication discrepancies grouped according to medication class and type of discrepancy. Data are number $(\%)$

\begin{tabular}{lrcrc}
\hline Medication Class & Medication Omitted & Wrong Dose or Frequency & Not Specified & Any Discrepancy \\
\hline Cardiovascular & $10(40)$ & 0 & $15(60)$ & $25(31)$ \\
Hormone-modifying & $4(40)$ & $1(10)$ & $5(50)$ & $10(12)$ \\
Hematologic/oncologic ${ }^{*}$ & $3(30)$ & 0 & $7(70)$ & $10(12)$ \\
CNS & $6(60)$ & $1(10)$ & $3(30)$ & $10(12)$ \\
Gastrointestinal & $3(75)$ & 0 & $1(25)$ & $4(5)$ \\
Other ${ }^{\dagger}$ & $4(18)$ & $1(5)$ & $17(77)$ & $22(27)$ \\
Total & $30(37)$ & $3(4)$ & $48(59)$ & $81(100)$ \\
\hline CNS = central nervous system. & & \\
$*$ \\
Includes chemotherapeutic agents or adjuvant therapies. \\
${ }^{\prime}$ Includes antimicrobial, musculoskeletal, dermatologic, autonomic, respiratory tract, and immune-modifying agents; antihistamines; vitamins/ \\
minerals; topical eye, ear, nose, and throat agents; and those not stated or known.
\end{tabular}




\section{Table IV}

Patient and hospitalization characteristics associated with medication discrepancies on admission: Logistic regression analysis

\begin{tabular}{|c|c|c|c|c|}
\hline Characteristic & Univariate Odds Ratio (95\% CI) & $P$ & Multivariate Odds Ratio (95\% CI) & $P$ \\
\hline Age, continuous (per 5-year increase) & $1.10(0.99-1.23)$ & 0.09 & $1.16(1.01-1.33)$ & 0.035 \\
\hline$<65 y^{*}$ & - & - & & \\
\hline $265 \mathrm{y}$ & $1.82(0.90-3.70)$ & 0.096 & & \\
\hline \multicolumn{5}{|l|}{ Sex } \\
\hline Male & $1.03(0.51-2.07)$ & 0.94 & & \\
\hline Female ${ }^{*}$ & - & - & & \\
\hline \multicolumn{5}{|l|}{ Race } \\
\hline White & $1.21(0.56-2.61)$ & 0.63 & & \\
\hline Black or other* & - & - & & \\
\hline No. of comorbid conditions & $1.03(0.90-1.18)$ & 0.65 & & \\
\hline Admitting service & & $0.036^{\dagger}$ & & \\
\hline Cardiology ${ }^{*}$ & - & - & & \\
\hline General medicine & $1.62(0.64-4.06)$ & 0.31 & & \\
\hline General surgery & $3.21(1.29-7.98)$ & 0.012 & $3.31(1.40-7.87)$ & $<0.007$ \\
\hline No. of medications on admission & $1.03(0.96-1.11)$ & 0.42 & & \\
\hline Any high-risk medications on admission & $63.14(7.93-502.45)$ & $<0.001$ & $76.68(9.13-643.76)$ & $<0.001$ \\
\hline
\end{tabular}




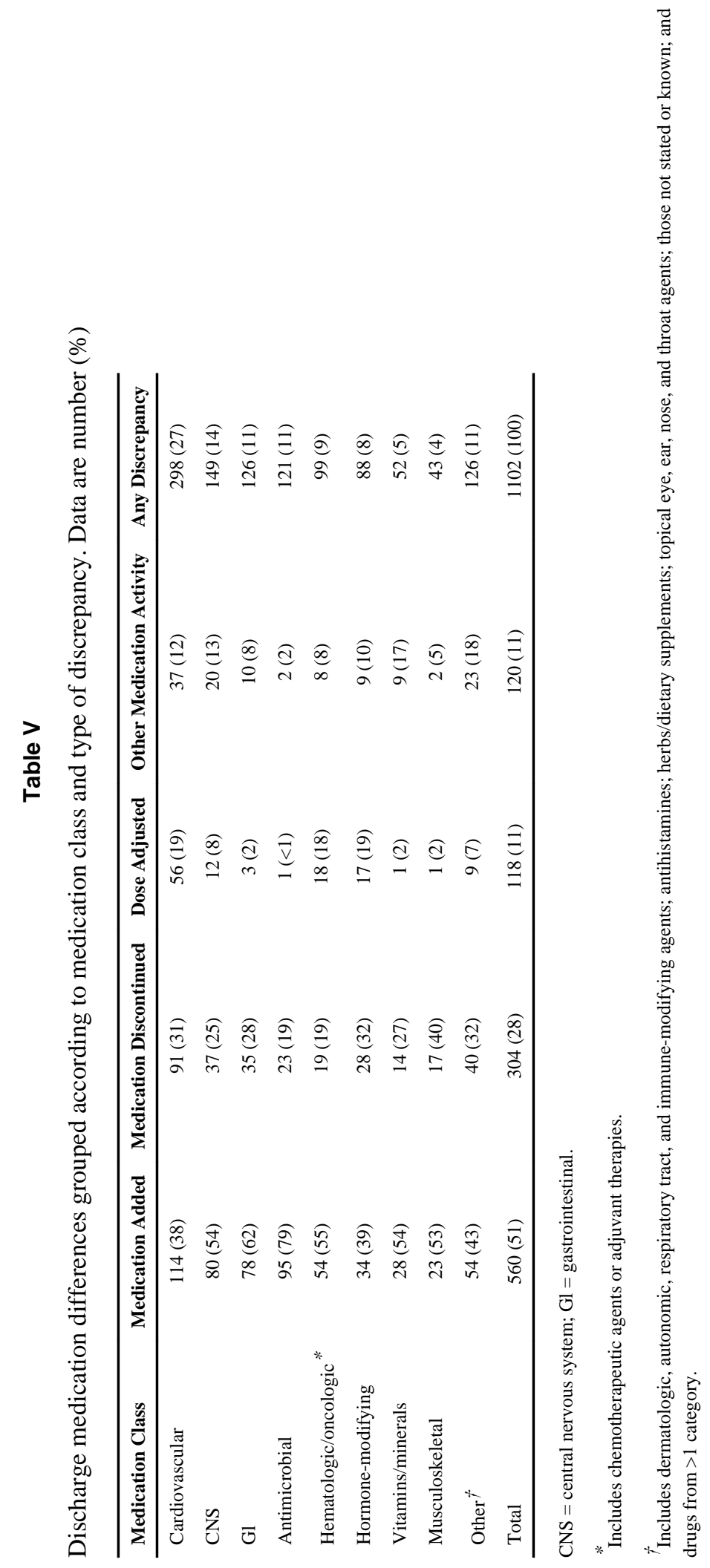




\section{Table VII}

Patient and hospitalization characteristics associated with medication differences at discharge: Logistic regression analysis

\begin{tabular}{|c|c|c|}
\hline Characteristic & Univariate Odds Ratio (95\% CI) & $P$ \\
\hline Age, continuous (per 5-year increase) & $1.02(0.82-1.28)$ & 0.83 \\
\hline \multicolumn{3}{|l|}{ Age,y } \\
\hline$<65^{*}$ & - & - \\
\hline 265 & $0.83(0.18-3.80)$ & 0.81 \\
\hline \multicolumn{3}{|l|}{ Sex } \\
\hline Male & $1.00(0.22-4.59)$ & 0.99 \\
\hline Female ${ }^{*}$ & - & - \\
\hline \multicolumn{3}{|l|}{ Race } \\
\hline White & $0.76(0.14-4.00)$ & 0.74 \\
\hline Black or other ${ }^{*}$ & - & - \\
\hline No. of comorbid conditions & $0.90(0.68-1.19)$ & 0.45 \\
\hline Admitting service & & $0.65^{t}$ \\
\hline Cardiology ${ }^{*}$ & - & - \\
\hline General medicine & $1.00(0.20-5.13)$ & 0.99 \\
\hline General surgery & $2.78(0.28-27.4)$ & 0.38 \\
\hline Length of stay & $1.70(0.97-3.00)$ & 0.065 \\
\hline No. of medications on admission & $1.01(0.85-1.20)$ & 0.89 \\
\hline No. of medications at discharge & $1.12(0.90-1.38)$ & 0.31 \\
\hline Any high-risk medications at discharge & $9.47(1.12-80.22)$ & 0.039 \\
\hline
\end{tabular}

